\title{
Morlet Wavelets in Quantum Mechanics
}

\author{
John Ashmead \\ School of Engineering and Applied Science, University of Pennsylvania, Philadelphia, Pennsylvania, United States. \\ E-mail:john.ashmead@timeandquantummechanics.com
}

Editors: Boaz Tamir, José R. Croca \& Danko Georgiev

Article history: Submitted on June 30, 2012; Accepted on September 5, 2012; Published on November 15, 2012.

\section{W} avelets offer significant advantages for the analysis of problems in quantum mechanics. Because wavelets are localized in both time and frequency they avoid certain subtle but potentially fatal conceptual errors that can result from the use of plane wave or $\delta$ function decomposition. Morlet wavelets in particular are well-suited for this work: as Gaussians, they have a simple analytic form and they work well with Feynman path integrals. But to take full advantage of Morlet wavelets we need to supply an explicit form for the inverse Morlet transform and a manifestly covariant form for the fourdimensional Morlet wavelet. We construct both here. Quanta 2012; 1: 58-70.

\section{Introduction}

Wavelet transforms represent a natural development of Fourier transforms and may be used for similar purposes. Where the Fourier transform lets us decompose a wave function into its component plane waves, a wavelet transform lets us decompose a wave function into its component wavelets. If we think of the plane waves as corresponding to pure tones, we may think of the wavelets as corresponding to the notes produced by physical instruments: of finite duration and spanning a finite range of

(c) $(9)$ This is an open access article distributed under the terms of the Creative Commons Attribution License CC-BY-3.0, which permits unrestricted use, distribution, and reproduction in any medium, provided the original author and source are credited. tones. Wavelets have two advantages over plane waves. First, they are localized in time and frequency. This can make them a better fit to the wave forms found in nature, which are always localized in both time and frequency. As a result, wavelet series will often converge faster than corresponding Fourier series. Second, there are many different wavelets to choose from. Therefore, we can tailor our wavelets to our problem. These advantages have resulted in their application to a wide variety of practical problems in acoustics, astronomy, medical imaging, computer graphics, meteorology, and so on. Morlet's original references are [1,2]. Wavelets are discussed in Chui, Meyer, and other texts [3-9]. Wavelets also have significant if less numerous applications on the theory side: canonical quantization of the electromagnetic field using a discrete wavelet basis [10], analysis of localization properties of photons using windowed wavelets [11], regularization of Euclidean field theories [12], and use of wavelets to provide Lorentz covariant, singularity-free, finite energy, zero action, localized solutions to the wave equation [13].

Wavelets offer significant benefits for the study of foundational questions in quantum mechanics as well. We will focus here specifically on Morlet wavelets. These are Gaussians, so are both easy to work with and a natural fit to path integrals [14-21], which typically consist of long series of Gaussian integrations. Use of Morlet wavelets can let us (1) avoid any need to invoke the problematic collapse of the wave function in the analysis of the Stern-Gerlach experiment, (2) avoid the use of artificial convergence factors or Wick rotation in computing path integrals, and (3) compute path integrals in a time 
symmetric way. But to prepare Morlet wavelets for their new responsibilities we need to (1) supply an explicit form for the 'admissibility constant' that is needed to define the inverse Morlet transform, and (2) provide a manifestly covariant extension of Morlet wavelets to four dimensions.

\section{Three applications of Morlet wavelets}

\subsection{Analyzing the Stern-Gerlach experiment}

In the original Stern-Gerlach experiment [22-24] a beam of silver atoms is sent through an inhomogeneous magnetic field. The beam is split into two: those atoms with spin up getting a kick in one direction; those with spin down in the opposite. This was striking first because it demonstrated the existence of spin and secondly because a classical system would have shown a continuous range of values for the spin, not just up and down. This split is regarded as a classic demonstration of the measurement problem, explained in the Copenhagen interpretation [25] as a collapse of the wave function into up and down components.

In the Stern-Gerlach experimental the initial wave function is typically modeled as the product of a plane wave and a spin vector. Replacing the plane waves with Gaussian test functions provides a more physically realistic model. Gondran and Gondran [26] have looked at the time evolution of such Gaussian wave functions in a SternGerlach apparatus. They show that when the wave function is modeled with Gaussian test functions, the spin up and spin down components split without any need to invoke a collapse. It works a bit like a diffraction experiment: there is coherent interference at two spots, incoherent at the rest. One may think of this as an internal diffraction effect.

Gondran and Gondran intended their work at least partly in support of the Bohm interpretation; however the math is independent of the interpretation. The implication is that - at least in this case - there is no need to invoke the highly problematic [27-30] collapse of the wave function.

The use of a single Gaussian test function is not of itself general. But with the use of the Morlet wavelet transform we can write an arbitrary square-integrable wave function as a sum over Gaussian test functions, making the Gondran and Gondran approach completely general.

To be sure, we could attempt to restore the honor of the plane wave by arguing that we could build up a Gaus- sian test function as a sum over such. But then why not eliminate the middleman and start with Gaussian test functions?

There are several related analyses of the Stern-Gerlach effect. Cruz-Barrios and Gómez-Camacho [31, 32] argue that we can explain the effect by modeling the atom with coherent internal states, whereas Venugopalan and coauthors [33-35] argue the effect is a result of decoherence. The Gondran and Gondran result is simpler in that it posits no additional structure (coherent internal states) or additional interaction (decoherence); standard quantum mechanics of its own suffices.

\subsection{Ensuring convergence of path integrals}

Morlet wavelets can assist in establishing convergence of Feynman path integrals without recourse to convergence factors as used in [14,15] or Wick rotation as in [20]; convergence of the slice-by-slice integrals in the path integral is a side-effect of the initial wave function being composed of Gaussians, for which convergence is automatic. It is sufficient to examine the free case.

We start with the free Schrödinger equation:

$$
\imath \frac{d}{d \tau} \psi_{\tau}(\vec{x})=-\frac{1}{2 m} \nabla^{2} \psi_{\tau}(\vec{x})
$$

The path integral expression for the kernel is given by:

$$
\begin{gathered}
K_{\tau}\left(\vec{x} ; \vec{x}^{\prime}\right)=\lim _{N \rightarrow \infty}\left(\frac{m}{2 \pi \imath \hbar \varepsilon}\right)^{\frac{3 N}{2}} \\
\times \int d \vec{x}_{1} \ldots d \vec{x}_{N-1} e^{\frac{\imath \varepsilon}{\hbar} \frac{m}{2} \sum_{j=0}^{N-1}\left(\frac{\vec{x}_{j+1}-\vec{x}_{j}}{\varepsilon}\right)^{2}}
\end{gathered}
$$

A typical integral is:

$K_{j}\left(\vec{x}_{j+1} ; \vec{x}_{j-1}\right)=\left(\frac{m}{2 \pi \imath \hbar \varepsilon}\right)^{\frac{3}{2}} \int d \vec{x}_{j} e^{\frac{\ell \varepsilon}{\hbar} \frac{m}{2}\left[\left(\frac{\vec{x}_{j+1}-\vec{x}_{j}}{\varepsilon}\right)^{2}+\left(\frac{\vec{x}_{j}-\vec{x}_{j-1}}{\varepsilon}\right)^{2}\right]}$

where $\varepsilon \equiv \frac{\tau}{N}$ is the width of a time slice.

The typical integral does not converge. We can force convergence by adding a small imaginary part $l \sigma$ to the mass: $m \rightarrow m+\imath \sigma$. Equivalently we could add a small imaginary part to the time step: $\varepsilon \rightarrow \varepsilon-\imath \sigma$. Or we could rotate time in the complex plane: $t \rightarrow t t$.

Now, focus attention on the first step:

$$
\psi_{1}\left(\vec{x}_{1}\right)=\int d \vec{x}_{0} K_{1}\left(\vec{x}_{1} ; \vec{x}_{0}\right) \psi_{0}\left(\vec{x}_{0}\right)
$$

Assume the initial wave function is a Gaussian:

$$
\psi_{1}\left(\vec{x}_{1}\right)=\left(\frac{m}{2 \pi i \hbar \varepsilon}\right)^{\frac{3}{2}}\left(\pi \sigma^{2}\right)^{-\frac{3}{4}} \int d \vec{x}_{0} e^{\frac{l \varepsilon}{\hbar} \frac{m}{2}\left(\frac{\overrightarrow{1}_{1}-\vec{x}_{0}}{\varepsilon}\right)^{2}-\frac{\left(\vec{x}_{0}-\left\langle\vec{x}_{0}\right\rangle\right)^{2}}{2 \sigma^{2}}}
$$


This integral is convergent of itself. The result is a (slightly wider) Gaussian. We can do an infinite series of these, with the initial wave function showing an increasing amount of middle-aged spread but with all of the integrals converging.

As an arbitrary wave function may be written, via Morlet wavelets, as a sum over Gaussian test functions, we have convergence in the general case, without the introduction of artificial convergence factors.

\subsection{Summing path integrals in a time symmetric way}

One immediate benefit of not needing convergence factors or Wick rotation is that we can treat time in a more symmetric way. One case where we might want to do this is in setting up a path integral analysis of the StückelbergSchrödinger equation:

$$
l \frac{d \psi_{u}(x)}{d u}=H \psi_{u}(x)
$$

Here $u$ is a formal parameter, a scalar of some kind perhaps the particle's proper time - and $H$ is a Lorentz invariant Hamiltonian. There are examples in Feynman [36, 37] and more recently in work by Land, Horwitz, and Seidewitz [21,38,39]. This approach has been sufficiently interesting that there are regular conferences held by the International Association for Relativistic Dynamics (IARD) devoted to this and related questions.

In the free case $H$ might be given by:

$$
\begin{gathered}
H=-\frac{1}{2 m}\left(l \frac{\partial}{\partial x^{\mu}}\right)\left(l \frac{\partial}{\partial x_{\mu}}\right) \\
=\frac{1}{2 m}\left(\frac{\partial^{2}}{\partial t^{2}}-\frac{\partial^{2}}{\partial x^{2}}-\frac{\partial^{2}}{\partial y^{2}}-\frac{\partial^{2}}{\partial z^{2}}\right)
\end{gathered}
$$

Note that because of the Lorentz invariance the time and space parts enter into $H$ with opposite sign, so in the path integral will have a problem converging in a Lorentz covariant way.

Path integral form for the kernel:

$$
\begin{aligned}
K_{\tau}\left(x ; x^{\prime}\right)= & \lim _{N \rightarrow \infty}\left(\frac{m}{2 \pi \sqrt{\imath} \hbar \varepsilon}\right)^{2 N} \int d t_{1} d \vec{x}_{1} \ldots d t_{N-1} d \vec{x}_{N-1} \\
& \times e^{-\frac{l \varepsilon}{\hbar} \frac{m}{2} \sum_{j=0}^{N-1}\left[\left(\frac{t_{j+1}-t_{j}}{\varepsilon}\right)^{2}-\left(\frac{\vec{x}_{j+1}-\vec{x}_{j}}{\varepsilon}\right)^{2}\right]}
\end{aligned}
$$

The pre-factor for the time part is the complex conjugate of the pre-factor for the space part:

$$
\left(\frac{m}{2 \pi \imath \hbar \varepsilon}\right)^{\frac{1}{2}} \rightarrow\left(\frac{\imath m}{2 \pi \hbar \varepsilon}\right)^{\frac{1}{2}}
$$

A typical slice:

$$
\begin{array}{r}
K_{j}\left(\vec{x}_{j+1} ; \vec{x}_{j-1}\right)=\left(\frac{m}{2 \pi \sqrt{i} \hbar \varepsilon}\right)^{2} \int d \vec{x}_{j} \\
\times e^{-\frac{l \varepsilon}{\hbar} \frac{m}{2}\left[\left(\frac{t_{j+1}-t_{j}}{\varepsilon}\right)^{2}-\left(\frac{\vec{x}_{j+1}-\vec{x}_{j}}{\varepsilon}\right)^{2}+\left(\frac{t_{j}-t_{j-1}}{\varepsilon}\right)^{2}-\left(\frac{\vec{x}_{j}-\vec{x}_{j-1}}{\varepsilon}\right)^{2}\right]}
\end{array}
$$

But now the addition of a small imaginary part to mass or time fails; any change that causes the time integrals to converge will cause the space integrals to diverge and vice versa. If we use different signs for time and space, then we break covariance.

Wick rotation fails for the same reason. Here we rotate time in the complex plane: $t \rightarrow \pm t t$. But if we pick one sign, the integral over the past will diverge; the other, the integral over the future.

Again, look at the first step:

$$
\psi_{1}\left(t_{1}, \vec{x}_{1}\right)=\int d t_{0} d \vec{x}_{0} K_{1}\left(t_{1}, \vec{x}_{1} ; t_{0}, \vec{x}_{0}\right) \psi_{0}\left(t_{0}, \vec{x}_{0}\right)
$$

Assume our initial wave function is given by a Gaussian test function:

$$
\begin{gathered}
\psi_{1}\left(t_{1}, \vec{x}_{1}\right)=\left(\frac{m}{2 \pi \sqrt{i} \hbar \varepsilon}\right)^{2} \frac{1}{\pi \sigma^{2}} \int d t_{0} d \vec{x}_{0} \\
\times e^{-\frac{\imath \varepsilon}{\hbar} \frac{m}{2}\left[\left(\frac{t_{1}-t_{0}}{\varepsilon}\right)^{2}-\left(\frac{\vec{x}_{1}-\vec{x}_{0}}{\varepsilon}\right)^{2}\right]-\frac{t_{0}^{2}+\left(\vec{x}_{0}-\left\langle\vec{x}_{0}\right\rangle\right)^{2}}{2 \sigma^{2}}}
\end{gathered}
$$

Now the integrals converge step by step. As any squareintegrable wave function may be written as a sum over such (see below) we have convergence. Of course to do this, we need covariant Morlet wavelets (see further below).

In many cases, an asymmetric treatment of time is harmless. But if we are analyzing time itself, then we do not want to wire the assumption that it is asymmetric into the maths. To do so would result in circular reasoning. The use of small imaginary factors or Wick rotation will not work for an analysis that is of time itself, as such approaches implicitly prejudge the conclusion.

\section{Morlet wavelets in one dimension}

We will first review the Morlet wavelet transform, then show how to compute the inverse Morlet wavelet transform explicitly.

To generate a set of wavelets we start with a mother wavelet $\phi(t)$. We get the general wavelet $\phi_{s l}(t)$ by scaling the mother wavelet by a scale factor $s$ and displacing her by a displacement $l$ :

$$
\phi_{s l}(t) \equiv|s|^{-\frac{1}{2}} \phi\left(\frac{t-l}{s}\right)
$$




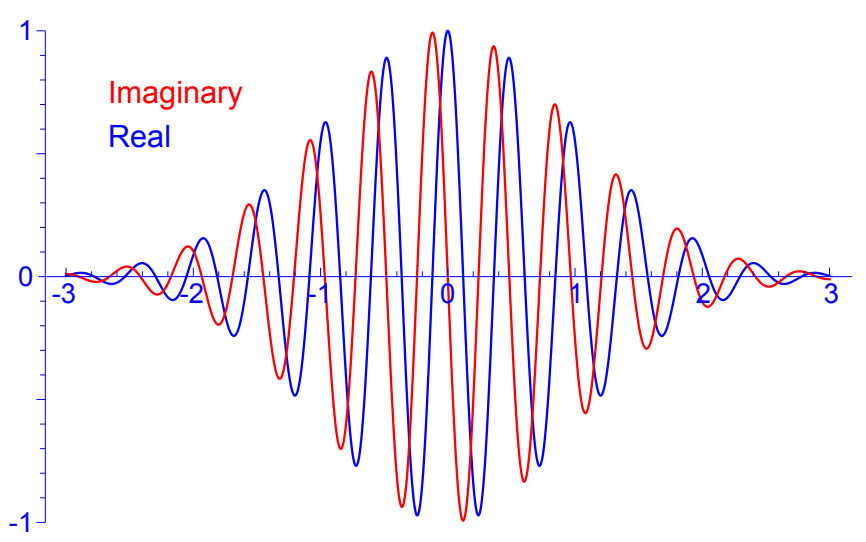

Figure 1: Real and imaginary parts of a mother Morlet wavelet $\phi(t)$ given by Equation 14 with $f=13$.

For Morlet wavelets the mother wavelet is given by:

$$
\phi(t)=\left(e^{-l f t}-e^{-\frac{1}{2} f^{2}}\right) e^{-\frac{1}{2} t^{2}}
$$

The second term is needed to satisfy the admissibility condition, discussed below. The parameter $t$ is often the time and $f$ may then be thought of as a reference frequency.

In some practical applications (i.e. [40]) the second term is dropped. However it is needed in general to ensure convergence of the inverse Morlet wavelet transform. The exact value of $f$ does not matter in principle, provided it is non-zero. If $f$ is zero, the mother wavelet is zero (and useless). We keep $f$ a variable to help in calculating the value of the admissibility constant $C_{f}$.

The general Morlet wavelet is created from the mother Morlet wavelet by scaling by $s$ and displacing by $l$ :

$$
\phi_{S l}(t) \equiv|s|^{-\frac{1}{2}}\left(e^{-l f\left(\frac{t-l}{s}\right)}-e^{-\frac{1}{2} f^{2}}\right) e^{-\frac{1}{2}\left(\frac{t-l}{s}\right)^{2}}
$$

Both scale $s$ and displacement $l$ run from $-\infty$ to $\infty$. A negative scale $s$ gives the complex conjugate of the Morlet wavelet with positive scale:

$$
\phi_{-s, l}(t)=\phi_{s, l}^{*}(t)
$$

The mother Morlet wavelet herself is given in this notation as the Morlet wavelet with scale factor one, displacement zero:

$$
\phi(t)=\phi_{1,0}(t)
$$

Any square integrable function $\psi$ may be expressed as a sum over Morlet wavelets. In principle this excludes $\delta$ functions and plane waves. We will see below they are handled correctly however. The Morlet wavelet transform of a wave function $\psi$ is given by:

$$
\tilde{\psi}_{s l}=\int_{-\infty}^{\infty} d t \phi_{s l}^{*}(t) \psi(t)
$$

We get the original $\psi$ back by integrating over the displacement and scale:

$$
\psi(t)=\frac{1}{C_{f}} \int_{-\infty}^{\infty} \frac{d s}{s^{2}} \int_{-\infty}^{\infty} d l \phi_{s l}(t) \tilde{\psi}_{s l}
$$

The admissibility constant is given by an integral over the square of the Fourier transform of the mother wavelet:

$$
C_{f} \equiv 2 \pi \int_{-\infty}^{\infty} \frac{d \omega}{|\omega|}|\hat{\phi}(\omega)|^{2}
$$

In the general case we could use a different set of wavelets for the forward and the inverse transforms; it is one of the attractions of Morlet wavelets that we do not need to do this.

The wavelet decomposition fails if $C_{f}$ is not finite. For $C_{f}$ to be finite, we see we need the zero frequency component of the Fourier transform of the Morlet wavelet mother to be zero:

$$
\hat{\phi}(0)=0 \Rightarrow \int_{-\infty}^{\infty} d t \phi(t)=0
$$

The Fourier transform of the Morlet mother is:

$$
\hat{\phi}(\omega)=\left(e^{f \omega}-1\right) e^{-\frac{1}{2}\left(f^{2}+\omega^{2}\right)}
$$

By inspection, we see the zero frequency component is zero. As noted above, the second term of the Morlet mother wavelet was included precisely to ensure this.

The Fourier transform of the general Morlet wavelet is:

$$
\hat{\phi}_{s l}(\omega)=|s|^{\frac{1}{2}} e^{l l \omega}\left(e^{f s \omega}-1\right) e^{-\frac{1}{2}\left(f^{2}+s^{2} \omega^{2}\right)}
$$

It may be written in terms of the Fourier transform of the mother:

$$
\hat{\phi}_{s l}(\omega)=|s|^{\frac{1}{2}} e^{l l \omega} \hat{\phi}(s \omega)
$$

\subsection{Normalization}

Morlet wavelets are not wave functions, but do not object to being treated as such. Their normalization is independent of their scale and displacement:

$$
\int d t \phi_{s l}^{*}(t) \phi_{s l}(t)=\sqrt{\pi}\left(e^{-f^{2}}-2 e^{-\frac{3}{4} f^{2}}+1\right)
$$


We can therefore write normalized Morlet wavelets as: $\phi_{s l}^{(\text {norm })}(t)=\left[\sqrt{\pi}\left(e^{-f^{2}}-2 e^{-\frac{3}{4} f^{2}}+1\right)\right]^{-\frac{1}{2}} \phi_{s l}(t)$

\subsection{Resolution of unity}

We can establish the completeness of the wavelet transform by very general methods, see [5].

But if we are only concerned with Morlet wavelets, we can take advantage of their specific character to give a less general but more immediate proof.

If we substitute the integral for $\tilde{\psi}_{s l}$ Equation 18) in the integral for the inverse Morlet wavelet transform (Equation 19p we get:

$$
\psi(t)=\frac{1}{C_{f}} \int_{-\infty}^{\infty} \frac{d s d l d t^{\prime}}{s^{2}} \phi_{s l}(t) \phi_{s l}^{*}\left(t^{\prime}\right) \psi\left(t^{\prime}\right)
$$

This will be true if we have:

$$
\delta\left(t-t^{\prime}\right)=\frac{1}{C_{f}} \int_{-\infty}^{\infty} \frac{d s d l}{s^{2}} \phi_{s l}(t) \phi_{s l}^{*}\left(t^{\prime}\right)
$$

This looks like a familiar decomposition in terms of a set of states weighted by $s^{-2}$. If we can show this directly, we will have a resolution of unity. To do this, we define the integral:

$$
I\left(t, t^{\prime}\right) \equiv \frac{1}{C_{f}} \int_{-\infty}^{\infty} \frac{d s d l}{s^{2}} \phi_{s l}(t) \phi_{s l}^{*}\left(t^{\prime}\right)
$$

We wish to show that this integral gives the $\delta$ function. We write the Morlet wavelets in terms of their Fourier transforms to get:

$$
\begin{aligned}
I\left(t, t^{\prime}\right)= & \frac{1}{C_{f}} \int_{-\infty}^{\infty} \frac{d s d l}{s^{2}} \int \frac{d \omega}{\sqrt{2 \pi}} e^{-l \omega t} \hat{\phi}_{s l}(\omega) \\
& \times \int \frac{d \omega^{\prime}}{\sqrt{2 \pi}} e^{\imath \omega^{\prime} t^{\prime}} \hat{\phi}_{s l}^{*}\left(\omega^{\prime}\right)
\end{aligned}
$$

Then we write the Fourier transforms of the wavelets in terms of the Fourier transform of the mother wavelet Equation 24:

$$
\begin{aligned}
& \frac{1}{C_{f}} \int_{-\infty}^{\infty} \frac{d s d l}{|s|} \int \frac{d \omega}{\sqrt{2 \pi}} e^{-l \omega(t-l)} \hat{\phi}(s \omega) \\
& \quad \times \int \frac{d \omega^{\prime}}{\sqrt{2 \pi}} e^{\imath \omega^{\prime}\left(t^{\prime}-l\right)} \hat{\phi}^{*}\left(s \omega^{\prime}\right)
\end{aligned}
$$

We recognize the integral over $l$ as a $\delta$ function in $\omega$ and $\omega^{\prime}$.

$$
\int \frac{d l}{2 \pi} e^{l\left(\omega-\omega^{\prime}\right) l}=\delta\left(\omega-\omega^{\prime}\right)
$$

We use this hitherto disguised $\delta$ function to do the integral over $\omega^{\prime}$.

$$
\frac{1}{C_{f}} \iint \frac{d s d \omega}{|s|} e^{-\imath \omega t} e^{\imath \omega t^{\prime}} \hat{\phi}(s \omega) \hat{\phi}^{*}(s \omega)
$$

We break the integral up into positive and negative $s$ parts:

$$
\begin{gathered}
\frac{1}{C_{f}} \int_{0}^{\infty} \frac{d s}{s} \int_{-\infty}^{\infty} d \omega e^{-l \omega t} e^{\imath \omega t^{\prime}} \hat{\phi}(s \omega) \hat{\phi}^{*}(s \omega) \\
+\frac{1}{C_{f}} \int_{-\infty}^{0} \frac{d s}{|s|} \int_{-\infty}^{\infty} d \omega e^{-l \omega t} e^{\imath \omega t^{\prime}} \hat{\phi}(s \omega) \hat{\phi}^{*}(s \omega)
\end{gathered}
$$

In the second term, replace $s$ by $-s$ and flip the sense of the integration:

$$
\begin{array}{r}
\frac{1}{C_{f}} \int_{0}^{\infty} \frac{d s}{s} \int_{-\infty}^{\infty} d \omega e^{-l \omega t} e^{l \omega t^{\prime}} \hat{\phi}(s \omega) \hat{\phi}^{*}(s \omega) \\
+\frac{1}{C_{f}} \int_{0}^{\infty} \frac{d s}{s} \int_{-\infty}^{\infty} d \omega e^{-l \omega t} e^{l \omega t^{\prime}} \hat{\phi}(-s \omega) \hat{\phi}^{*}(-s \omega)
\end{array}
$$

We change the variable of integration to $s^{\prime}=s \omega$, then combine the two terms:

$\frac{1}{C_{f}} \int_{0}^{\infty} \frac{d s^{\prime}}{s^{\prime}} \int_{-\infty}^{\infty} d \omega e^{-l \omega t} e^{l \omega t^{\prime}}\left[\hat{\phi}\left(s^{\prime}\right) \hat{\phi}^{*}\left(s^{\prime}\right)+\hat{\phi}\left(-s^{\prime}\right) \hat{\phi}^{*}\left(-s^{\prime}\right)\right]$

We identify the $\omega$ integration as still another $\delta$ function, one which can come outside of the integrals:

$$
2 \pi \delta\left(t-t^{\prime}\right) \frac{1}{C_{f}} \int_{0}^{\infty} \frac{d s^{\prime}}{s^{\prime}}\left[\hat{\phi}\left(s^{\prime}\right) \hat{\phi}^{*}\left(s^{\prime}\right)+\hat{\phi}\left(-s^{\prime}\right) \hat{\phi}^{*}\left(-s^{\prime}\right)\right]
$$

We replace $s^{\prime}$ by $-s^{\prime}$ in the second term: 


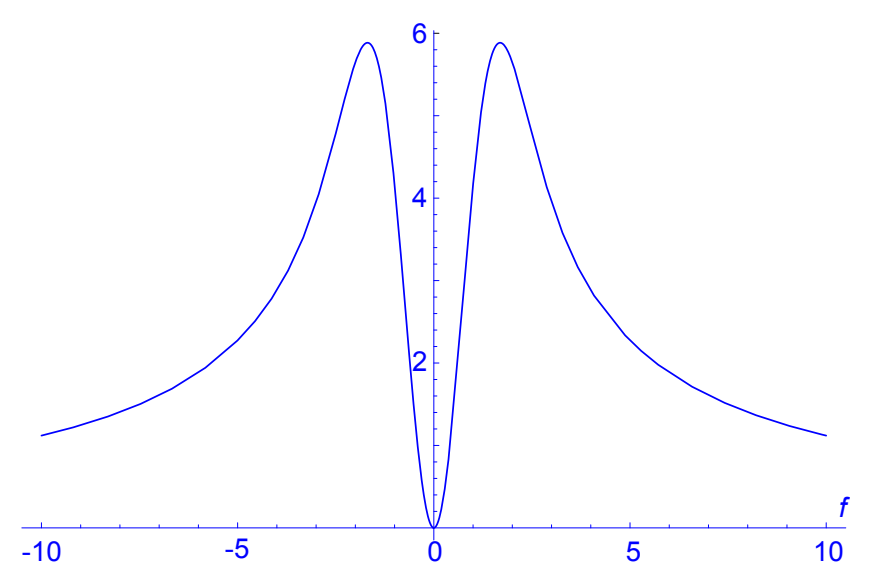

Figure 2: The admissibility constant $C_{f}$ as a function of $f$.

$$
\begin{aligned}
& \int_{0}^{\infty} \frac{d s^{\prime}}{s^{\prime}}\left[\hat{\phi}\left(-s^{\prime}\right) \hat{\phi}^{*}\left(-s^{\prime}\right)\right] \\
& =-\int_{-\infty}^{0} \frac{d s^{\prime}}{s^{\prime}}\left[\hat{\phi}\left(s^{\prime}\right) \hat{\phi}^{*}\left(s^{\prime}\right)\right] \\
& =\int_{-\infty}^{0} \frac{\mathrm{d} s^{\prime}}{\left|s^{\prime}\right|}\left[\hat{\phi}\left(s^{\prime}\right) \hat{\phi}^{*}\left(s^{\prime}\right)\right]
\end{aligned}
$$

Giving for the integral:

$$
\int_{-\infty}^{\infty} \frac{d s^{\prime}}{\left|s^{\prime}\right|} \hat{\phi}\left(s^{\prime}\right) \hat{\phi}^{*}\left(s^{\prime}\right)
$$

Which is $\frac{C_{f}}{2 \pi}$ (see Equation 20) so we have, as required:

$$
I\left(t, t^{\prime}\right)=\delta\left(t-t^{\prime}\right)
$$

\subsection{Calculation of admissibility constant}

To actually use the inverse Morlet transform we need an explicit expression for the value of the admissibility constant. By substituting the Fourier transform of the mother Morlet wavelet (Equation 22) in the formula for the admissibility constant Equation 20 we get:

$$
C_{f}=2 \pi e^{-f^{2}} \int_{-\infty}^{\infty} \frac{d \omega}{|\omega|}\left(e^{\omega f}-1\right)^{2} e^{-\omega^{2}}
$$

For convenience, we define a new integral:

$$
I(f) \equiv \int_{-\infty}^{\infty} \frac{d \omega}{|\omega|}\left(e^{\omega f}-1\right)^{2} e^{-\omega^{2}}
$$

For $f=0$, we find $I(f)=0$ by inspection. This is expected given that the original mother wavelet is zero when $f$ is zero. As $\omega$ goes to zero, the integrand goes as $f^{2} \omega$ so is well-behaved in the small $\omega$ limit. And as $\omega$ goes to $\infty$ the integrand goes as $\frac{e^{2 f \omega-\omega^{2}}}{|\omega|}$ so is also wellbehaved in the large $\omega$ limit. Therefore we can write $I(f)$ as:

$$
I(f)=\int_{0}^{f} d f \frac{d I(f)}{d f}
$$

The advantage of taking the derivative with respect to $f$ is that it gets rid of the troubling factor of $|\omega|$ in the denominator. We break up the integral over $\omega$ into negative and positive parts:

$$
I(f)=\int_{-\infty}^{0} \frac{\mathrm{d} \omega}{|\omega|}\left(e^{\omega f}-1\right)^{2} e^{-\omega^{2}}+\int_{0}^{\infty} \frac{\mathrm{d} \omega}{|\omega|}\left(e^{\omega f}-1\right)^{2} e^{-\omega^{2}}
$$

then change variables from $\omega \rightarrow-\omega$ in the negative part to get:

$$
I(f)=\int_{0}^{\infty} \frac{d \omega}{|\omega|}\left[\left(e^{-\omega f}-1\right)^{2}+\left(e^{\omega f}-1\right)^{2}\right] e^{-\omega^{2}}
$$

The derivative of $I$ with respect to $f$ is:

$$
\frac{d I(f)}{d f}=2 \int_{0}^{\infty} d \omega\left[\left(e^{2 \omega f}-e^{-2 \omega f}\right)-\left(e^{\omega f}-e^{-\omega f}\right)\right] e^{-\omega^{2}}
$$

which can be re-written as:

$$
\frac{d I(f)}{d f}=4 \int_{0}^{\infty} d \omega[\sinh (2 \omega f)-\sinh (\omega f)] e^{-\omega^{2}}
$$

After integration with respect to $\omega$ we have:

$$
\frac{d I(f)}{d f}=2 \sqrt{\pi}\left[e^{f^{2}} \operatorname{erf}(f)-e^{\frac{1}{4} f^{2}} \operatorname{erf}\left(\frac{f}{2}\right)\right]
$$

We integrate this with respect to $f$ to get a pair of generalized hypergeometric functions ${ }_{2} F_{2}$ :

$$
I(f)=f^{2}\left[2{ }_{2} F_{2}\left(1,1 ; \frac{3}{2}, 2 ; f^{2}\right)-{ }_{2} F_{2}\left(1,1 ; \frac{3}{2}, 2 ; \frac{f^{2}}{4}\right)\right]
$$

Therefore, we have for $C_{f}$ : 


$$
\begin{aligned}
C_{f}= & 2 \pi e^{-f^{2}} f^{2}\left[2_{2} F_{2}\left(1,1 ; \frac{3}{2}, 2 ; f^{2}\right)\right. \\
& \left.-{ }_{2} F_{2}\left(1,1 ; \frac{3}{2}, 2 ; \frac{f^{2}}{4}\right)\right]
\end{aligned}
$$

For $f=1$ we obtain $C_{1} \approx 4.1636$. This can be checked by doing the original integral numerically, see subsection 6.2.

For small $f, C_{f}$ goes as:

$$
\lim _{f \rightarrow 0} C_{f} \rightarrow 2 \pi f^{2}
$$

For large $f, C_{f}$ goes as:

$$
\lim _{f \rightarrow \infty} C_{f} \rightarrow e^{-f^{2}}
$$

At this point we have an explicit form for the inverse Morlet transform, so have reached our objective. We now apply the Morlet wavelet transform to some interesting cases.

\subsection{Gaussian test functions}

Gaussian test functions (squeezed states) are the most important case:

$$
\psi_{\sigma E \tau}(t)=\left(\pi \sigma^{2}\right)^{-\frac{1}{4}} e^{-l E(t-\tau)-\frac{(t-\tau)^{2}}{2 \sigma^{2}}}
$$

The Fourier transform of this Gaussian test function is:

$$
\hat{\psi}_{\sigma E \tau}(\omega)=\left(\frac{\sigma^{2}}{\pi}\right)^{\frac{1}{4}} e^{i \omega \tau-\frac{(E-\omega)^{2} \sigma^{2}}{2}}
$$

\subsubsection{Analysis}

Per (Equation 18), the Morlet wavelet transform of a Gaussian test function is:

$$
\tilde{\psi}_{s l}^{(\sigma E \tau)}=\int_{-\infty}^{\infty} \mathrm{d} t \phi_{s l}^{*}(t) \psi_{\sigma E \tau}(t)
$$

By inspection, we can write the Morlet wavelet in the transform as the sum of two Gaussians:

$$
\phi_{s l}^{*}(t)=\pi^{\frac{1}{4}}\left(\psi_{s \frac{f}{s} l}^{*}(t)-e^{-\frac{f^{2}}{2}} \psi_{s 0 l}^{*}(t)\right)
$$

This means the transform reduces to a pair of Gaussian integrations:

$$
\begin{aligned}
\tilde{\psi}_{s l}^{(\sigma E \tau)}= & \int_{-\infty}^{\infty} d t^{\prime}|s|^{-\frac{1}{2}}\left[e^{l f \frac{\left(t^{\prime}-l\right)}{s}}-e^{-\frac{f^{2}}{2}}\right] e^{-\frac{1}{2}\left(\frac{t^{\prime}-l}{s}\right)^{2}} \\
& \times\left(\pi \sigma^{2}\right)^{-\frac{1}{4}} e^{-l E\left(t^{\prime}-\tau\right)-\frac{\left(t^{\prime}-\tau\right)^{2}}{2 \sigma^{2}}}
\end{aligned}
$$

The integral is elementary, giving:
The Morlet transform looks like a sum of inner products of Gaussians:

$$
\tilde{\psi}_{s l}^{(\sigma E \tau)}=\pi^{\frac{1}{4}}\left(\left\langle\psi_{s \frac{f}{s} l} \mid \psi_{\sigma E \tau}\right\rangle-e^{-\frac{f^{2}}{2}}\left\langle\psi_{s 0 l} \mid \psi_{\sigma E \tau}\right\rangle\right)
$$

This suggests (looking just at the leading term) that the greatest contributions to the transform will come when $s \sim \sigma, \frac{f}{s} \sim E$, and $l \sim \tau$.

\subsubsection{Inverse Morlet wavelet transform}

We expect the original Gaussian function will be recovered by (Equation 19):

$$
\psi_{\sigma E \tau}(t)=\frac{1}{C_{f}} \int_{-\infty}^{\infty} \frac{d s d l}{s^{2}} \phi_{s l}(t) \tilde{\psi}_{s l}^{(\sigma E \tau)}
$$

Without loss of generality, we simplify by assuming that $\tau=0$ in the original Gaussian test function and write the Morlet wavelet as the sum of a pair of Gaussians:

$$
\begin{gathered}
\psi_{\sigma E}(t)=\frac{1}{C_{f}} \int_{-\infty}^{\infty} \frac{d s d l}{s^{2}}\left(\frac{2 \sqrt{\pi} \sigma}{s^{2}+\sigma^{2}}\right)^{\frac{1}{2}}\left[e^{-l f\left(\frac{t-l}{s}\right)}-e^{-\frac{f^{2}}{2}}\right] e^{-\frac{1}{2}\left(\frac{t-l}{s}\right)^{2}} \\
\times\left[e^{-l \frac{f s l}{s^{2}+\sigma^{2}}-\frac{1}{2} \frac{(E s-f)^{2} \sigma^{2}}{s^{2}+\sigma^{2}}}-e^{-\frac{f^{2}}{2}-\frac{1}{2} \frac{(E s \sigma)^{2}}{s^{2}+\sigma^{2}}}\right] e^{-l E \frac{\sigma^{2} l}{s^{2}+\sigma^{2}}-\frac{1}{2} \frac{l^{2}}{s^{2}+\sigma^{2}}}
\end{gathered}
$$

The integral over $l$ is straightforward, as all the terms are Gaussians in $l$ : 


$$
\begin{gathered}
\psi_{\sigma E}(t)=\frac{1}{C_{f}} \int_{-\infty}^{\infty} \frac{d s}{|s|} 2 \pi^{\frac{3}{4}}\left(\frac{\sigma}{2 s^{2}+\sigma^{2}}\right)^{\frac{1}{2}} \\
\times\left[e^{-\frac{2 E^{2} s^{2} \sigma^{2}+2 f^{2}\left(2 s^{2}+\sigma^{2}\right)+2 t E \sigma^{2} t+t^{2}}{2\left(2 s^{2}+\sigma^{2}\right)}}\right. \\
-2 e^{-\frac{3 f^{2} s^{2}+2 \sigma^{2}\left(f^{2}-f E s+E^{2} s^{2}\right)+2 t t\left(f s+E \sigma^{2}\right)+t^{2}}{2\left(2 s^{2}+\sigma^{2}\right)}} \\
\left.+e^{-\frac{2 \sigma^{2}(f-E s)^{2}+2 t t\left(2 f s+E \sigma^{2}\right)+t^{2}}{2\left(2 s^{2}+\sigma^{2}\right)}}\right]
\end{gathered}
$$

The limit of the integrand as $s$ goes to zero is:

$$
\begin{gathered}
2 e^{-f^{2}-\imath E t-\frac{t^{2}}{2 \sigma^{2}}} f^{2} \pi^{\frac{3}{4}} \sigma^{-\frac{9}{2}}|s| \\
\times\left(\sigma^{2}+E^{2} \sigma^{4}-2 \imath E \sigma^{2} t-t^{2}\right)+O\left[s^{2}\right]
\end{gathered}
$$

The limit as $s$ goes to $\pm \infty$ is:

$$
\pm \frac{\sqrt{2 \sigma} \pi \pi^{\frac{3}{4}} e^{-f^{2}-\frac{\mathrm{E}^{2} \sigma^{2}}{2}}\left(1-2 e^{\frac{1}{4} f^{2}}+e^{f^{2}}\right)}{s^{2}}+O\left[\frac{1}{s}\right]^{3}
$$

Our integral is therefore neither singular at the origin nor divergent at infinity. Of course, we expect this since we are guaranteed by the decomposition theorem that this integral will give the original Gaussian. To show explicitly we get the original Gaussian we take the Fourier transform of both sides, with respect to $t$. The simplification is dramatic - most of the factors come outside of the integral over $s$. On the left we have (Equation 24):

$$
\hat{\psi}_{\sigma E}(\omega)=\left(\frac{\sigma^{2}}{\pi}\right)^{\frac{1}{4}} e^{-\frac{(E-\omega)^{2} \sigma^{2}}{2}}
$$

On the right we get:

$$
\frac{1}{C_{f}} 2 \pi e^{-f^{2}}\left(\frac{\sigma^{2}}{\pi}\right)^{\frac{1}{4}} e^{-\frac{(E-\omega)^{2} \sigma^{2}}{2}} \int_{-\infty}^{\infty} \frac{d s}{|s|} e^{-s^{2} \omega^{2}}\left(e^{f s \omega}-1\right)^{2}
$$

We change variables in the integral $s^{\prime} \equiv s \omega$ :

$$
\left(\frac{\sigma^{2}}{\pi}\right)^{\frac{1}{4}} e^{-\frac{(E-\omega)^{2} \sigma^{2}}{2}} \frac{1}{C_{f}} 2 \pi e^{-f^{2}} \int_{-\infty}^{\infty} \frac{\mathrm{d} s^{\prime}}{\left|s^{\prime}\right|} 2 e^{-s^{\prime 2}}\left(e^{f s^{\prime}}-1\right)^{2}
$$

We note the integral is essentially the admissibility constant (Equation 43). Factors cancel yielding:

$$
\left(\frac{\sigma^{2}}{\pi}\right)^{\frac{1}{4}} e^{-\frac{(E-\omega)^{2} \sigma^{2}}{2}}
$$

Which is identical to the left hand side, as was to be shown.

\subsection{Other Applications}

We will compute the Morlet wavelet transforms of $\delta$ functions, plane waves, and - to achieve maximum selfreferentiality - a Morlet wavelet itself.

\subsection{1 $\delta$ functions}

Since the $\delta$ function is not a square-integrable function, we are not guaranteed the wavelet transform will work. We therefore write the $\delta$ function as a limit of Gaussian test functions:

$$
\delta(x)=\lim _{\sigma \rightarrow 0^{+}} \frac{1}{\sqrt{2 \pi} \sigma} e^{-\frac{x^{2}}{2 \sigma^{2}}}
$$

This lets us use the result for a Gaussian test function Equation 60:

$$
\begin{gathered}
\tilde{\delta}_{s l}(\tau)=\lim _{\sigma \rightarrow 0^{+}}\left(s^{2}+\sigma^{2}\right)^{-\frac{1}{2}}|s|^{\frac{1}{2}} \\
\times\left[e^{-l \frac{f s}{s^{2}+\sigma^{2}}(l-\tau)-\frac{1}{2} \frac{f^{2} \sigma^{2}}{s^{2}+\sigma^{2}}}-e^{-\frac{f^{2}}{2}}\right] e^{-\frac{1}{2} \frac{(l-\tau)^{2}}{s^{2}+\sigma^{2}}}
\end{gathered}
$$

Taking the limit as $\sigma$ goes to zero:

$$
\tilde{\delta}_{s l}(\tau)=|s|^{-\frac{1}{2}}\left[e^{-l f\left(\frac{l-\tau}{s}\right)}-e^{-\frac{f^{2}}{2}}\right] e^{-\frac{1}{2} \frac{(l-\tau)^{2}}{s^{2}}}
$$

This is itself a Morlet wavelet:

$$
\tilde{\delta}_{s l}(\tau)=\phi_{s l}^{*}(\tau)
$$

We get the same result by computing the Morlet wavelet transform directly:

$$
\begin{aligned}
& \tilde{\delta}_{s l}(\tau)=\int_{-\infty}^{\infty} d t^{\prime} \phi_{s l}^{*}\left(t^{\prime}\right) \delta\left(t^{\prime}-\tau\right) \\
& =|s|^{-\frac{1}{2}}\left(e^{l f \frac{\tau-l}{s}}-e^{-\frac{f^{2}}{2}}\right) e^{-\frac{1}{2}\left(\frac{\tau-l}{s}\right)^{2}}
\end{aligned}
$$

Since the demonstration of the resolution of unity only applies to square-integrable functions, we verify the inverse transform. We want to show:

$$
\delta(t-\tau)=\frac{1}{C_{f}} \int_{-\infty}^{\infty} \frac{d s d l}{s^{2}} \phi_{s l}(t) \phi_{s l}^{*}(\tau)
$$

However this is just what we showed when we computed the admissibility constant (Equation 20), so we are done. 


\subsubsection{Plane waves}

The Morlet wave transform of a plane wave:

$$
\chi_{E}(t) \equiv \frac{1}{\sqrt{2 \pi}} e^{-l E t}
$$

is given by:

$$
\begin{aligned}
\tilde{\chi}_{s l}(E) & =\int d t|s|^{-\frac{1}{2}}\left[e^{l f \frac{t-l}{s}}-e^{-\frac{f^{2}}{2}}\right] \\
& \times e^{-\frac{1}{2}\left(\frac{t-l}{s}\right)^{2}} \frac{1}{\sqrt{2 \pi}} e^{-l E t}
\end{aligned}
$$

The integral is essentially the Fourier transform of a Morlet wavelet:

$$
\tilde{\chi}_{s l}(E)=\hat{\phi}_{s l}^{*}(E)
$$

For the inverse transform to be valid we require:

$$
\begin{gathered}
\chi_{E}(t)=\frac{1}{C_{f}} \int \frac{d s d l}{s^{2}} \phi_{s l}(t) \tilde{\chi}_{s l}(E) \\
=\frac{1}{C_{f}} \int \frac{d s d l}{s^{2}} \phi_{s l}(t) \hat{\phi}_{s l}^{*}(E)
\end{gathered}
$$

To show this, we take the Fourier transform Equation 113 of each side. On the left side we get:

$$
\delta(\omega-E)
$$

On the right side we write the Fourier transforms of the Morlet wavelets (Equation 24) in terms of the Fourier transforms of their mothers (Equation 22):

$$
\frac{1}{C_{f}} \int \frac{d s d l}{s^{2}}|s|^{\frac{1}{2}} e^{l l \omega} \hat{\phi}(s \omega)|s|^{\frac{1}{2}} e^{-l l E} \hat{\phi}^{*}(s E)
$$

The integral over $l$ is a $\delta$ function, which we pull out of the integral, leaving the now familiar admissibility constant (Equation 20) behind:

$$
\frac{2 \pi}{C_{f}} \delta(\omega-E) \int \frac{d s}{|s|} \hat{\phi}(s \omega) \hat{\phi}^{*}(s \omega)=\delta(\omega-E)
$$

\subsubsection{Morlet wavelet transform of a Morlet wavelet}

We look at the Morlet wavelet transform of a Morlet wavelet Equation 15) with $\sigma E$ replacing $f$ and $\sigma$ replacing $s$ :

$$
\Phi_{\sigma E \tau}(t) \equiv|\sigma|^{-\frac{1}{2}}\left[e^{-l E(t-\tau)}-e^{-\frac{\sigma^{2} E^{2}}{2}}\right] e^{-\frac{1}{2}\left(\frac{t-\tau}{\sigma}\right)^{2}}
$$

Per (Equation 18), the Morlet wavelet transform is given by:

$$
\tilde{\Phi}_{s l}^{(\sigma E \tau)}=\int_{-\infty}^{\infty} d t^{\prime} \phi_{s l}^{*}(t) \Phi_{\sigma E \tau}(t)
$$

To apply the results for Gaussian test functions we split the incoming Morlet wavelet $\Phi_{\sigma E \tau}(t)$ into its two Gaussians then use the results for Gaussian test functions Equation 60) to read off the results:

$$
\tilde{\Phi}_{s l}^{(\sigma E \tau)}=\left(2 \pi \frac{\sigma}{s^{2}+\sigma^{2}}|s|\right)^{\frac{1}{2}}
$$

$$
\begin{aligned}
& \times\left\{\left[e^{-l \frac{f s}{s^{2}+\sigma^{2}}(l-\tau)-\frac{1}{2} \frac{(E s-f)^{2} \sigma^{2}}{s^{2}+\sigma^{2}}}-e^{-\frac{f^{2}}{2}-\frac{1}{2} \frac{(E s \sigma)^{2}}{s^{2}+\sigma^{2}}}\right] e^{-l E \frac{\sigma^{2}}{s^{2}+\sigma^{2}}(l-\tau)}\right. \\
& \left.-\left[e^{-l \frac{f s}{s^{2}+\sigma^{2}}(l-\tau)-\frac{1}{2} \frac{f^{2} \sigma^{2}}{s^{2}+\sigma^{2}}-\frac{\sigma^{2} E^{2}}{2}}-e^{-\frac{f^{2}}{2}-\frac{\sigma^{2} E^{2}}{2}}\right]\right\} e^{-\frac{1}{2} \frac{(l-\tau)^{2}}{s^{2}+\sigma^{2}}}
\end{aligned}
$$

\section{Covariant Morlet wavelets}

\subsection{Strategy}

We would like to generalize Morlet wavelets to four dimensions (one time, three space) in a way that is manifestly covariant. We will do this by taking the direct product of Morlet wavelets in time and the three space dimensions. The natural generalization of the Gaussian part of the one-dimensional Morlet wavelet is:

$$
e^{-\frac{1}{2} x^{2}} \rightarrow e^{-\frac{1}{2}\left(x_{\mu} x^{\mu}\right)}=e^{\frac{1}{2}\left(t^{2}-x^{2}-y^{2}-z^{2}\right)}
$$

This clearly diverges in $t$. We have to fix this without losing manifest covariance.

We will assume we start in a specific frame $M$, possibly the center-of-mass frame. We will define the fourdimensional Morlet wavelet as the product of four onedimensional Morlet wavelets, then write our results in a way that is Lorentz-invariant.

\subsection{Construction}

We take the four-dimensional mother Morlet wavelet as the direct product of four one-dimensional mother Morlet wavelets, one for each coordinate:

$$
\phi(t) \rightarrow \phi(t) \phi(x) \phi(y) \phi(z)
$$

We write the four dimensional mother Morlet wavelet as the product of four one-dimensional mother Morlet wavelets (Equation 14):

$$
\begin{gathered}
\phi(t, x, y, z)=\left(e^{-l f_{0} t}-e^{-\frac{f_{0}^{2}}{2}}\right)\left(e^{l f_{1} x}-e^{-\frac{f_{1}^{2}}{2}}\right) \\
\times\left(e^{l f_{2} y}-e^{-\frac{f_{2}^{2}}{2}}\right)\left(e^{l f_{3} z}-e^{-\frac{f_{3}^{2}}{2}}\right) e^{-\frac{t^{2}+x^{2}+y^{2}+z^{2}}{2}}
\end{gathered}
$$


By scaling and displacing each component separately we get:

$$
\begin{gathered}
\phi_{s l}(t, x, y, z)=\left|s_{0} s_{1} s_{2} s_{3}\right|^{-\frac{1}{2}}\left(e^{-l f_{0} \frac{t-l_{0}}{s_{0}}}-e^{-\frac{f_{0}^{2}}{2}}\right) \\
\times\left(e^{l f_{1} \frac{x-l_{1}}{s_{1}}}-e^{-\frac{f_{1}^{2}}{2}}\right)\left(e^{l f_{2} \frac{y-l_{2}}{s_{2}}}-e^{-\frac{f_{2}^{2}}{2}}\right)\left(e^{l f_{3} \frac{z-l_{3}}{s_{3}}}-e^{-\frac{f_{3}^{2}}{2}}\right) \\
\times e^{-\frac{1}{2}\left[\left(\frac{t-l_{0}}{s_{0}}\right)^{2}+\left(\frac{x-l_{1}}{s_{1}}\right)^{2}+\left(\frac{y-l_{2}}{s_{2}}\right)^{2}+\left(\frac{z-l_{3}}{s_{3}}\right)^{2}\right]}
\end{gathered}
$$

Using (Equation 22), the Fourier transform of the mother Morlet wavelet is:

$$
\begin{gathered}
\hat{\phi}\left(E, p_{x}, p_{y}, p_{z}\right)=\left(e^{f_{0} E}-1\right)\left(e^{f_{1} p_{x}}-1\right)\left(e^{f_{2} p_{y}}-1\right) \\
\times\left(e^{f_{3} p_{z}}-1\right) e^{-\frac{f_{0}^{2}+f_{1}^{2}+f_{2}^{2}+f_{3}^{2}}{2}-\frac{E^{2}+p_{x}^{2}+p_{y}^{2}+p_{z}^{2}}{2}}
\end{gathered}
$$

The Fourier transform of the general Morlet wavelet is:

$$
\begin{gathered}
\hat{\phi}_{s l}\left(E, p_{x}, p_{y}, p_{z}\right)=\left|s_{0} s_{1} s_{2} s_{3}\right|^{\frac{1}{2}} e^{l\left(l_{0} E-l_{1} p_{x}-l_{2} p_{y}-l_{3} p_{z}\right)} \\
\times\left(e^{s_{0} f_{0} E}-1\right)\left(e^{s_{1} f_{1} p_{x}}-1\right)\left(e^{s_{2} f_{2} p_{y}}-1\right)\left(e^{s_{3} f_{3} p_{z}}-1\right) \\
\times e^{-\frac{f_{0}^{2}+f_{1}^{2}+f_{2}^{2}+f_{3}^{2}}{2}-\frac{s_{0}^{2} E^{2}+s_{1}^{2} p_{x}^{2}+s_{2}^{2} p_{y}^{2}+s_{3}^{2} p_{z}^{2}}{2}}
\end{gathered}
$$

Now we have to promote various non-covariant bits to covariant bits.

The scale factors enter into the inverse Morlet integral in a slightly awkward way:

$$
\int \frac{d s_{0}}{s_{0}^{2}} \frac{d s_{1}}{s_{1}^{2}} \frac{d s_{2}}{s_{2}^{2}} \frac{d s_{3}}{s_{3}^{2}}
$$

The simplest approach to this is to treat the four scale factors as so many scalars.

The obvious choices for the displacement $l$ and the reference frequency $f$ are to treat them as four vectors. For the displacement a single four vector will suffice:

$$
l=\left(l_{0}, l_{1}, l_{2}, l_{3}\right)
$$

We will need one four vector for each reference frequency:

$$
\begin{aligned}
& F^{(0)} \equiv\left(f_{0}, 0,0,0\right) \\
& F^{(1)} \equiv\left(0, f_{1}, 0,0\right) \\
& F^{(2)} \equiv\left(0,0, f_{2}, 0\right) \\
& F^{(3)} \equiv\left(0,0,0, f_{3}\right)
\end{aligned}
$$

For convenience, we define the sum over all four $F$ 's as:

$$
F \equiv \sum_{n=0}^{3} F^{(n)}=\left(f_{0}, f_{1}, f_{2}, f_{3}\right)
$$

This is also a four vector. Note that the raw frequencies $f_{0}, f_{1}, f_{2}, f_{3}$ are themselves scalars since they are defined with respect to the specific frame $M$.

To represent the sums as Lorentz invariants we define a set of second rank tensors (with their inverses):

$$
\begin{gathered}
\Sigma_{\mu}^{(n) v} \equiv\left(\begin{array}{cccc}
s_{0}^{-n} & 0 & 0 & 0 \\
0 & -s_{1}^{-n} & 0 & 0 \\
0 & 0 & -s_{2}^{-n} & 0 \\
0 & 0 & 0 & -s_{3}^{-n}
\end{array}\right) \\
\left(\frac{1}{\Sigma^{(n)}}\right)_{\mu}^{v} \equiv\left(\begin{array}{cccc}
s_{0}^{n} & 0 & 0 & 0 \\
0 & -s_{1}^{n} & 0 & 0 \\
0 & 0 & -s_{2}^{n} & 0 \\
0 & 0 & 0 & -s_{3}^{n}
\end{array}\right)
\end{gathered}
$$

We need the explicit forms for $n$ from 0 to 2 :

$$
\begin{gathered}
\Sigma_{\mu}^{(0) v} \equiv\left(\begin{array}{cccc}
1 & 0 & 0 & 0 \\
0 & -1 & 0 & 0 \\
0 & 0 & -1 & 0 \\
0 & 0 & 0 & -1
\end{array}\right) \\
\Sigma_{\mu}^{(1) v} \equiv\left(\begin{array}{cccc}
s_{0}^{-1} & 0 & 0 & 0 \\
0 & -s_{1}^{-1} & 0 & 0 \\
0 & 0 & -s_{2}^{-1} & 0 \\
0 & 0 & 0 & -s_{3}^{-1}
\end{array}\right) \\
\Sigma_{\mu}^{(2) v} \equiv\left(\begin{array}{cccc}
s_{0}^{-2} & 0 & 0 & 0 \\
0 & -s_{1}^{-2} & 0 & 0 \\
0 & 0 & -s_{2}^{-2} & 0 \\
0 & 0 & 0 & -s_{3}^{-2}
\end{array}\right)
\end{gathered}
$$

The choice of signature $(1,-1,-1,-1)$ ensures convergence.

With these definitions the mother Morlet wavelet is:

$$
\phi\left(x_{\mu}\right)=\left\{\prod_{n=0}^{3}\left[e^{-l F^{(n) \mu} x_{\mu}}-e^{-\frac{1}{2} F^{(n) \mu} F_{\mu}}\right]\right\} e^{-\frac{1}{2} x^{\mu} \Sigma_{\mu}^{(0) v} x_{v}}
$$

and the general Morlet wavelet is:

$$
\begin{array}{r}
\phi_{\Sigma l}\left(x_{\mu}\right)=\sqrt{\operatorname{det}\left(\Sigma^{(1)}\right)} e^{-\frac{1}{2}\left(x^{\mu}-l^{\mu}\right) \Sigma_{\mu}^{(2) v}\left(x_{v}-l_{v}\right)} \\
\times\left[\prod_{n=0}^{3} e^{-l F^{(n) \mu} \Sigma_{\mu}^{(0) \sigma} \Sigma_{\varpi}^{(1) v}\left(x_{v}-l_{v}\right)}-e^{-\frac{1}{2} F^{(n) \mu} \Sigma_{\mu}^{(0) v} F_{v}^{(n)}}\right]
\end{array}
$$

While we have worked this out in frame $M$, as it is written in terms of covariant quantities it is valid in all frames. We have therefore guaranteed Lorentz covariance of the Morlet wavelets.

Note that the choice of frame defines a set of Morlet wavelets; with each frame there is a distinct set of Morlet wavelets. If we have multiple frames we wish to work with we will need to tag each Morlet wavelet with the 
frame it comes from. Usually there is an obvious choice of frame, i.e. the center-of-mass frame.

With these definitions, the Fourier transform of the mother Morlet wavelet is:

$$
\hat{\phi}(p)=\left\{\prod_{n=0}^{3}\left[e^{F^{(n)} \frac{1}{\Sigma^{(0)}} p}-1\right]\right\} e^{-F \frac{1}{2 \Sigma^{(0)}} F-\frac{1}{2} p \frac{1}{2 \Sigma(0)} p}
$$
is:

The Fourier transform of the general Morlet wavelet

$$
\begin{aligned}
\hat{\phi}_{\Sigma l}(p)= & \sqrt{\frac{1}{\operatorname{det}\left(\Sigma^{(1)}\right)}} e^{l p l-F \frac{1}{2 \Sigma^{(0)}} F-\frac{1}{2} p \frac{1}{2 \Sigma^{(2)}} p} \\
& \times\left\{\prod_{n=0}^{3}\left[e^{F^{(n)} \frac{1}{\Sigma^{(1)}} p}-1\right]\right\}
\end{aligned}
$$

\subsection{Resolution of unity}

Any square integrable function $\psi(t, x, y, z)$ may be expressed as a sum over these Morlet wavelets. The covariant Morlet wavelet transform is given by:

$$
\tilde{\psi}_{\Sigma l}=\int_{-\infty}^{\infty} d^{4} x \phi_{\Sigma l}^{*}(x) \psi(x)
$$

And the inverse is given by:

$$
\psi(x)=\frac{1}{C_{f_{0}} C_{f_{1}} C_{f_{2}} C_{f_{3}}} \int_{-\infty}^{\infty}\left(\prod_{n=0}^{3} \frac{d s_{n}}{\left|s_{n}\right|^{2}}\right) \int_{-\infty}^{\infty} d^{4} l \phi_{\Sigma l}(x) \tilde{\psi}_{\Sigma l}
$$

The resolution of unity and the values of the constants of admissibility follow directly from the results for one dimension.

The solutions for Gaussian test functions, $\delta$ functions, and plane waves are merely the direct products of the corresponding one-dimensional wave functions.

We have therefore reached our second and final objective: to generalize the Morlet wavelet transform to four dimensions in a way which is manifestly covariant.

\subsection{Alternative approaches}

Alternative (and more sophisticated) lines of attack are possible. For instance in [9] or in [41] two dimensional wavelets are generated from the mother wavelet by using displacements, rotations (in the $x y$ plane), and a single scale factor:

$$
\phi_{R l s}(x, y)=\frac{1}{s} \phi\left[\overleftrightarrow{R} \cdot \frac{(\vec{r}-\vec{l})}{s}\right]
$$

where $R$ is a rotation matrix (in two dimensions).
By analogy, we could generalize one-dimensional wavelets to four dimensions by using displacements $l$, Lorentz transformations:

$$
\phi_{\Lambda l s}\left(x_{\mu}\right)=\frac{1}{s^{2}} \phi\left[\frac{1}{s} \Lambda_{\mu}^{v}\left(x_{v}-l_{v}\right)\right]
$$

But establishing convergence, verifying the resolution of unity, and computing the admissibility constant for these wavelets would be a new project. Our immediate requirement is merely to establish that there is at least one set of covariant Morlet wavelets.

\section{Summary}

The naive use of plane wave or $\delta$ function decomposition can create artificial difficulties in the analysis of foundational questions of quantum mechanics. The use of Morlet wavelet decomposition avoids these difficulties. With the explicit calculation of the admissibility constant and the demonstration of covariant Morlet wavelets, we have eliminated two of the barriers to full use of this powerful technology for the analysis of foundational questions in quantum mechanics.

\section{Appendix}

\subsection{Conventions for the Fourier transform}

For the Fourier transform from time $t$ to frequency $\omega$ we are using:

$$
\hat{f}(\omega) \equiv \frac{1}{\sqrt{2 \pi}} \int_{-\infty}^{\infty} d t e^{i \omega t} f(t)
$$

with inverse Fourier transform:

$$
f(t)=\frac{1}{\sqrt{2 \pi}} \int_{-\infty}^{\infty} d \omega e^{-\imath \omega t} \hat{f}(\omega)
$$

In the Fourier transform in four dimensions time and space enter with opposite signs:

$$
\hat{f}(\omega, \vec{k}) \equiv \frac{1}{4 \pi^{2}} \int_{-\infty}^{\infty} d t d \vec{x} e^{l \omega t-l \vec{k} \cdot \vec{x}} f(t, \vec{x})
$$

with inverse Fourier transform:

$$
f(t, \vec{x})=\frac{1}{4 \pi^{2}} \int_{-\infty}^{\infty} d \omega d \vec{k} e^{-l \omega t+l \vec{k} \cdot \vec{x}} \hat{f}(\omega, \vec{k})
$$




\subsection{Direct calculation of the admissibility constant}

To check the results for the admissibility constant Equation 43, we ran a numeric calculation of the values of $f$ from $-3 \pi \rightarrow 3 \pi$. The integrand: $|\omega|^{-1}\left(e^{f \omega}-1\right)^{2} e^{-\omega^{2}}$ is real, smooth, and positive definite, making it a perfect candidate for numerical integration. Numerical integration produced results visually indistinguishable from the formula for $C_{f}$ given by Equation 52. On a test of 100 evenly spaced points from $-3 \pi \rightarrow 3 \pi$, with $f=1$, the maximum error was $2.72 \times 10^{-8}$ and the average absolute error was $8.2 \times 10^{-10}$. These residuals are easily explained in terms of rounding.

Much of the work here was done using the program Wolfram's Mathematica (version 7). However a certain amount of care is need when using this tool. An attempt to have it compute directly the integral for $I(f)$ given by Equation 44 produces the expression given in Equation 51 plus five additional terms: $2 \pi \operatorname{erfi}\left(\frac{1}{2}|f|\right)-$ $\pi \operatorname{erfi}(|f|)+\ln (-f)+\ln (f)-\ln \left(f^{2}\right)$. This is incorrect by inspection: the three $\ln$ terms add a value of $\ln (-1)=\imath \pi$ which is nonsense, given that the original integral is real. The other terms are real so offer no escape. The usual moral in working with Mathematica or any math software applies: as Walter Donovan, the villain in Indiana Jones and the Last Crusade, said to Indiana Jones: trust no one.

\section{References}

[1] Morlet J, Arens G, Fourgeau E, Glard D. Wave propagation and sampling theory. Part I: Complex signal and scattering in multilayered media. Geophysics 1982; 47 (2): 203-221. http://dx.doi.org/10. $1190 / 1.1441328$

[2] Morlet J, Arens G, Fourgeau E, Giard D. Wave propagation and sampling theory. Part II: Sampling theory and complex waves. Geophysics 1982; 47 (2): 222-236. http://dx.doi.org/10.1190/1. 1441329

[3] Chui CK. An Introduction to Wavelets. Wavelet Analysis and Its Applications, vol.1, Boston: Academic Press, 1992.

[4] Meyer Y. Wavelets and Operators. Cambridge Studies in Advanced Mathematics, Cambridge: Cambridge University Press, 1992.

[5] Kaiser G. A Friendly Guide to Wavelets. Boston: Birkhäuser, 1994.

[6] van den Berg JC. Wavelets in Physics. Cambridge: Cambridge University Press, 1999.
[7] Addison PS. The Illustrated Wavelet Transform Handbook: Introductory Theory and Applications in Science, Engineering, Medicine and Finance. Bristol: Institute of Physics Publishing, 2002.

[8] Bratteli O, Jørgensen PET. Wavelets through a Looking Glass: The World of the Spectrum. Applied and Numerical Harmonic Analysis, Boston: Birkhäuser, 2002.

[9] Antoine J-P, Murenzi R, Vandergheynst P, Ali ST. Two-Dimensional Wavelets and their Relatives. Cambridge: Cambridge University Press, 2004. http://dx.doi.org/10.1017/ CB09780511543395

[10] Havukainen M. Wavelets as basis functions in canonical quantization, 2000. http://arxiv, org/abs/quant-ph/0006083

[11] Kim YS. Wavelets and information-preserving transformations, 1996. http://arxiv.org/abs/ quant-ph/9610018

[12] Altaisky MV. Wavelet based regularization for Euclidean field theory, 2003. http://arxiv.org/ abs/hep-th/0305167

[13] Visser M. Physical wavelets: Lorentz covariant, singularity-free, finite energy, zero action, localized solutions to the wave equation. Physics Letters A 2003; 315 (3-4): 219-224. http://arxiv.org/ abs/hep-th/0304081

[14] Feynman RP, Hibbs AR. Quantum Mechanics and Path Integrals. New York: McGraw-Hill Companies, 1965.

[15] Schulman LS. Techniques and Applications of Path Integration. New York: John Wiley \& Sons, 1981.

[16] Swanson MS. Path Integrals and Quantum Processes. New York: Academic Press, 1992.

[17] Khandekar DC, Lawande SV, Bhagwat KV. PathIntegral Methods and Their Applications. Singapore: World Scientific Publishing Company, 1993.

[18] Marchewka A, Schuss Z. Path-integral approach to the Schrödinger current. Physical Review A 2000; 61 (5): 052107. http://arxiv.org/abs/ quant-ph/9903076

[19] Kleinert H. Path Integrals in Quantum Mechanics, Statistics, and Polymer Physics, and Financial Markets. Singapore: World Scientific Publishing Company, 2004. 
[20] Zinn-Justin J. Path Integrals in Quantum Mechanics. Oxford Graduate Texts, Oxford: Oxford University Press, 2005.

[21] Seidewitz E. Foundations of a spacetime path formalism for relativistic quantum mechanics. Journal of Mathematical Physics 2006; 47 (11): 112302-112329. http://arxiv.org/abs/ quant-ph/0507115

[22] Gerlach W, Stern O. Der experimentelle Nachweis des magnetischen Moments des Silberatoms. Zeitschrift für Physik 1922; 8 (1): 110-111. http: //dx.doi.org/10.1007/BF01329580

[23] Gerlach W, Stern O. Der experimentelle Nachweis der Richtungsquantelung im Magnetfeld. Zeitschrift für Physik A Hadrons and Nuclei 1922; 9 (1): 349-352. http://dx.doi.org/10.1007/ BF01326983

[24] Gerlach W, Stern O. Das magnetische Moment des Silberatoms. Zeitschrift für Physik 1922; 9 (1): 353-355. http://dx.doi.org/10.1007/ BFQ1326984

[25] von Neumann J. Mathematical Foundations of Quantum Mechanics. Investigations In Physics, Princeton: Princeton University Press, 1955.

[26] Gondran M, Gondran A. A complete analysis of the Stern-Gerlach experiment using Pauli spinors, 2005. http://arxiv.org/abs/quant-ph/0511276

[27] Schrödinger E. Die gegenwärtige Situation in der Quantenmechanik. Naturwissenschaften 1935; 23 (48): 807-812. http://dx.doi.org/10.1007/ BF01491891

[28] Schrödinger E. Die gegenwärtige Situation in der Quantenmechanik. Naturwissenschaften 1935; 23 (49): 823-828. http://dx.doi.org/10.1007/ BF01491914

[29] Schrödinger E. Die gegenwärtige Situation in der Quantenmechanik. Naturwissenschaften 1935; 23 (50): 844-849. http://dx.doi.org/10.1007/ BFQ1491987

[30] Schrödinger E. The present situation in quantum mechanics: a translation of Schrödinger's 'cat paradox' paper. In: Quantum Theory and Measurement, Wheeler JA, Zurek WH (editors), New Jersey: Princeton University Press, 1983, pp.152-167.
[31] Cruz-Barrios S, Gómez-Camacho J. Semiclassical description of scattering with internal degrees of freedom. Nuclear Physics A 1998; 636 (1): 70-84. http://dx.doi.org/10.1016/ S0375-9474(98)00176-6

[32] Cruz-Barrios S, Gómez-Camacho J. Semiclassical description of Stern-Gerlach experiments. Physical Review A 2000; 63 (1): 012101. http://arxiv. org/abs/quant-ph/0010079

[33] Venugopalan A, Kumar D, Ghosh R. Analysis of the Stern-Gerlach measurement, 1995. http://arxiv. org/abs/quant-ph/9501022

[34] Venugopalan A. Decoherence and Schrödinger-cat states in a Stern-Gerlach-type experiment. Physical Review A 1997; 56 (5): 4307-4310. http://dx. doi.org/10.1103/PhysRevA.56.4307

[35] Venugopalan A. Pointer states via decoherence in a quantum measurement. Physical Review A 1999; 61 (1): 012102. http://arxiv.org/abs/ quant-ph/9909005

[36] Feynman RP. Mathematical formulation of the quantum theory of electromagnetic interaction. Physical Review 1950; 80 (3): 440-457. http://dx.doi. org/10.1103/PhysRev.80.440

[37] Feynman RP. An operator calculus having applications in quantum electrodynamics. Physical Review 1951; 84 (1): 108-128. http://dx.doi.org/10. 1103/PhysRev. 84.108

[38] Land MC, Horwitz LP. Off-shell quantum electrodynamics, 1996. http://arxiv.org/abs/hep-th/ 9601021

[39] Horwitz LP. Second quantization of the Stueckelberg relativistic quantum theory and associated gauge fields, 1998. http://arxiv.org/abs/ hep-th/9804155

[40] Johnson RW. Symmetrization and enhancement of the continuous Morlet transform. International Journal of Wavelets, Multiresolution and Information Processing 2012; 10 (1): 1250009. http://arxiv. org/abs/0912.1126

[41] Perel MV, Sidorenko MS. New physical wavelet 'Gaussian wave packet'. Journal of Physics A: Mathematical and Theoretical 2007; 40 (13): 3441-3461. http://arxiv.org/abs/math-ph/0701051 